\title{
All-Optical Multipoint-to-Point Routing in WDM Mesh Networks
}

\author{
Fen Zhou • Miklós Molnár • Bernard Cousin
}

Received: date / Accepted: date

\begin{abstract}
In this article, the routing and wavelength assignment (RWA) problem for supporting multipointto-point communications in all-optical WDM mesh networks is investigated. Two efficient algorithms, namely Reverse Shortest Path Tree routing (RSPT) and $k$ Bounded Edge Disjoint Path routing (EDPR), are proposed. We proved that the problem of minimizing the total cost while establishing a multipoint-to-point session can be solved in polynomial time of $O(|V| \log |V|+$ $|V|+|E|)$ by the RSPT algorithm, where $|V|$ and $|E|$ denote the number of nodes and the number of edges in the network respectively. Nevertheless, the solution provided by the EDPR algorithm produces a significant reduction in the maximum number of wavelengths required per link (i.e., the link stress) for a multipointto-point session compared to RSPT algorithm. EDPR algorithm can also approximate to the optimal total cost with a ratio of $k$. Simulations are done to assess these two algorithms. Numerical results demonstrate their efficiencies in supporting multipoint-to-point communications in all-optical WDM networks.
\end{abstract}

A preliminary version of part of this work was presented in [14] at IEEE LCN 2009, Zürich.

Fen Zhou, Miklós Molnár, and Bernard Cousin

IRISA, Campus de Beaulieu, Rennes 35042, France

Tel.: +33-299842537

Fax: +33-299847171

E-mail: \{fen.zhou, molnar, bernard.cousin\}@irisa.fr

Fen Zhou, Miklós Molnár

INSA de Rennes, 20 Avenue des Buttes de Cöesmes, Rennes 35043, France

Bernard Cousin

University of Rennes 1, Campus de Beaulieu, Rennes 35042, France
Keywords WDM Networks · Multipoint-to-point Communication · Routing and Wavelength Assignment (RWA) · Light-startree · Reverse Shortest Path Tree $(\mathrm{RSPT}) \cdot$ Edge Disjoint Path Routing (EDPR)

All-optical Wavelength Division Multiplexing (WDM) networks, which are built on the concept of wavelength routing, have been deployed in the Internet infrastructure for decades to satisfy increasing demand for bandwidth [1]. As optical crossconnects (OXCs) become mature and commercially available, it enables a light beam to pass an intermediate optical node without the opticalelectrical-optical (OEO) conversion. By widely employing optical OXCs, all-optical WDM networks overcome the electro-optic bottleneck [2] and thus are gifted at providing huge bandwidth, low signal attenuation and distortion as well as favorable transmission delay [3]. Relying on inherent advantages, WDM networks are capable of supporting bandwidth-driven multimedia services in the Internet, such as VoIP, video conference, online community-based communications (e.g., blog, voting), etc. It broadly consists of four traffic patterns: point-to-point communications (or unicast), point-tomultipoint communications (multicast), multipoint-topoint communications (MP2P) and multipoint-to-multipoint (MP2MP) communications.

To support unicast communications in all-optical WDM networks, lightpaths are introduced to reduce the number of hops that a packet has to traverse [4]. A lightpath is a set of consecutive wavelength channels on the same wavelength, which connects a transmitter at a source node to a receiver at a destination node.

To satisfy the multicast services in all-optical WDM networks, the lightpath is extended to a light-tree [4] to minimize the number of transceivers and the average hop distance for given traffic demands. A light-tree can 
be viewed as a point-to-multipoint generalization of a lightpath. But, in order to support multicast, the optical switches should be equipped with optical splitters to split the light signal into several branches. In the case of sparse splitting [5], where only a small fraction of network nodes are capable of splitting, the light-forest [6] concept is introduced, which was focused by many researches $[7,15,16]$. To improve the multicast routing again and save more fiber channel cost, a new structure called light-hierarchy is investigated in [13]. Different from the light-tree, the light-hierarchy permits cycles in the multicast structure and thus overcomes the inherent drawback of the light-tree.

For multipoint-to-multipoint case, the core-based tree (CBT, $[8,9]$ ) structure could be applied with some proper modifications. In a core-based tree structure, a core node is employed to collect messages from all the source nodes and then forward the collected messages to all the destination nodes.

Supporting multipoint-to-point communications is also important and indispensable for wavelength-routed WDM networks. To the best of our knowledge, no other literature has addressed this problem before except our work in progress study [14]. Therefore, in this paper we extend our previous work and go deeply into the routing and wavelength assignment (RWA) problem for multipoint-to-point communications in all-optical WDM networks. The following assumptions are made in our study (i) the number of wavelengths supported per fiber link is sufficient to establish a multipoint-to-point communication, (ii) wavelength converters are not available due to the high cost and complex architecture, (iii) the optical traffic grooming switches $[10,11]$, which enable to aggregate low speed traffic streams into one high capacity wavelength, are also unavailable because of the lack of intelligent network control and automatic provisioning functionality [11].

An optical multipoint-to-point communication generally involves a simultaneous light signal transmission from several source nodes to the same sink node. Multipoint-to-point communication is the reverse of multicasat. However, they work totally differently in all-optical WDM networks. For multicasting, the optical switches should be capable of light splitting so that they can diffuse the same light signal to several direct predecessors simultaneously. While for multipoint-to-point communication, the optical switches receive different light signals from the source nodes, hence either the switches should groom the light signals emitted by different sources into one and forward it on one wavelength, or they have to forward the incoming light signals of different sources with each one on a different wavelength. Unfortunately, the optical traffic groom-

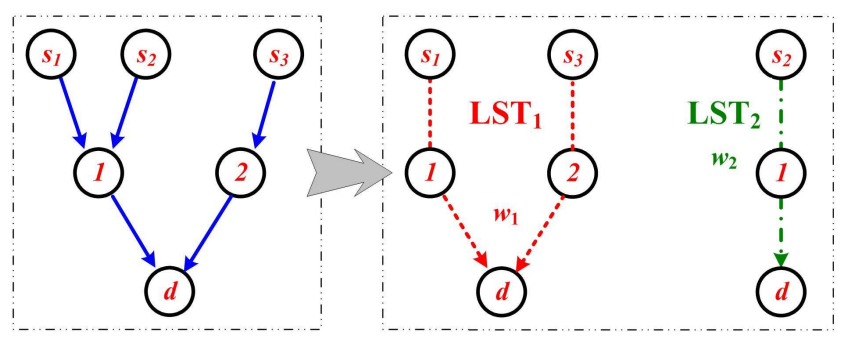

Fig. 1 An example of light-startrees

ing switches are not available, and thus the optical switches perform in the latter way. In all-optical WDM networks, two basic physical constraints are always encountered. The first one, namely wavelength continuity constraint, implies the same wavelength should be retained along a lightpath without wavelength conversion. And the other one, called the distinct wavelength constraint, does not permit to assign the same wavelength for two lightpaths sharing a common fiber link. For multipoint-to-point communications in all-optical networks, the distinct wavelength constraint is even more strict. The light signals emitted by two source nodes can be carried on the same wavelength only if the lightpaths from these two source nodes to the same sink node are edge disjoint. Thereby, we introduce a light-startree structure to route a multipoint-to-point communication. A light-startree is consisted of several edge disjoint lightpaths terminated at the same sink node but assigned only one wavelength. An example of light-startrees is given in Fig. 1. In the left dashdotted rectangle, a multipoint-to-point communication $m p 2 p\left(\left(s_{1}, s_{2}, s_{3}\right), d\right)$ arrives. Two light-startrees in the right dash-dot rectangle (i.e., $L S T_{1}$ is assigned wavelength $w_{1}$ and $L S T_{2}$ is assigned wavelength $w_{2}$ ) can be used to route this session.

Based on the light-startree structure, two efficient routing and wavelength assignment (RWA) algorithms are presented in our paper. The proposed Reverse Shortest Path Tree routing algorithm is proven to be able to minimize the number of wavelength channels used (i.e., the total cost). Besides, it can solve the routing and wavelength assignment problem for a multipointto-point session in polynomial time of $O(|V| \log |V|+$ $|V|+|E|)$, where $|V|$ and $|E|$ denote the number of nodes and the number of edges in the network respectively. To reduce the maximum number of wavelengths required per link (i.e., link stress) by a multipoint-topoint communication request, a $k$-Bounded Edge Disjoint Path routing algorithm is introduced. It is proven to provide a $k$-approximation of the total cost and require a favorable link stress for a multipoint-to-point session. Extensive simulations are done to assess the performance of these two algorithms. 
The rest of this paper is organized as follows. Section 1 formulates the routing and wavelength assignment problem of multipoint-to-point communications in all-optical WDM networks. Two routing and wavelength assignment algorithms are introduced in Section 2. The performance of these two algorithms are evaluated by mathematical analysis in Section 3. Numerical results are obtained by simulation in Section 4 . Finally, a summary of this paper is given in Section 5 .

\section{Multipoint-to-point Communications in All-Optical WDM Networks}

The problem of routing and wavelength assignment (RWA) for multipoint-to-point communications is studied in all-optical WDM networks. A WDM network can be modeled by an undirected graph $G(V, E, c, W)$. $V$ denotes the set of nodes in the network. Each edge $e \in E$ is associated with a weight function $c(e)$, which signifies the cost of fiber link channel $e . c(e)$ is additive along a lightpath $\operatorname{LP}(u, v)$, where $u$ and $v$ are two end point nodes of the lightpath. $W$ denotes the number of wavelengths that could be supported in a fiber link. We consider a multipoint-to-point session $\operatorname{mp} 2 p(S, d)$, which requires to set up simultaneous lightpaths from each source $s_{i} \in S$ to the same destination $d$. Due to the distinct wavelength constraint in WDM networks, two lightpaths could be assigned the same wavelength only if they are edge disjoint. Hence, the multipoint-topoint communication can be realized either by an individual unicast path to the destination for each source or a set of light-startrees. A light-startree is a set of edge-disjoint lightpaths, which share the same receiver end point and occupy the same wavelength. One lightstartree may not be sufficient to span all the source nodes of a multipoint-to-point session, and thus several light-startrees may be used with each on a different wavelength. To carry out a multipoint-to-point session, a RWA algorithm should try to save as much wavelength channel cost as possible while overcoming the optical constraints. The total cost of a multipointto-point communication can be calculated by the sum of fiber link channel cost in the set of light-startrees (LST) computed out. i.e.,

$c[m p 2 p(S, d)]=\sum_{L S T_{i} \in m p 2 p(S, d)} \sum_{e \in L S T_{i}} c(e)$

Besides this, the maximum number of wavelengths required per link for a multipoint-to-point session (i.e., link stress) is also important for WDM networks. This is because, physically, the number of wavelengths supported on a fiber link is limited to a fixed number. If

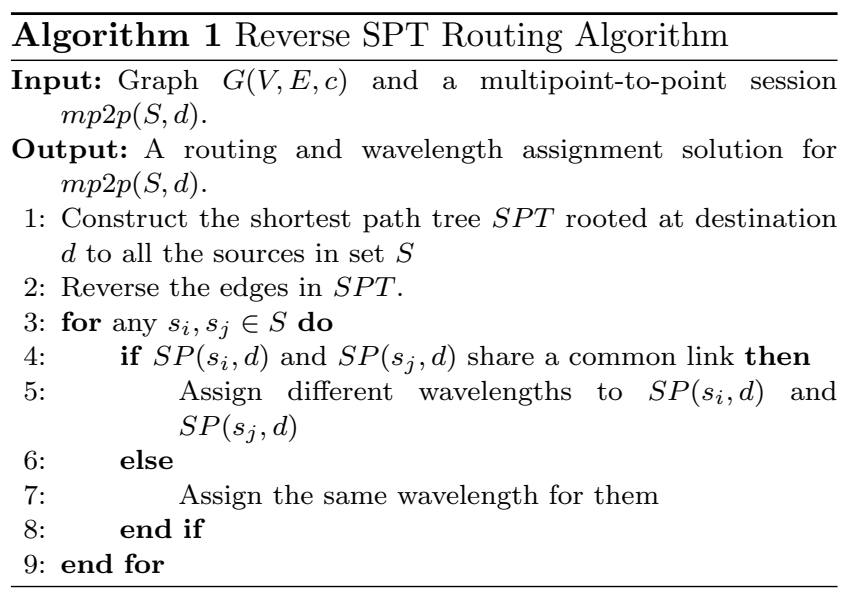

a multipoint-to-point session spends too many wavelengths in one fiber link, then the number of wavelengths left for other sessions will be limited, which thus results in the congestion of some links. Hence, balancing the multipoint-to-point traffic and avoiding the blockade of communications should also be taken into account by an efficient routing algorithm. Generally, the set of light-startrees computed out for a multipoint-topoint session are not edge disjoint and thus the link stress equals the number of light-startrees and at most the number of source nodes in a multipoint-to-point session.

\section{Proposed Solutions}

In this section, we propose two routing and wavelength assignment algorithms for multipoint-to-point communications in all-optical WDM networks. The first one, namely Reverse Shortest Path Tree routing algorithm, tries to optimize the total cost for a session. The second one, namely $k$-Bounded Edge Disjoint Path Routing algorithm (EDRP), is primarily designated to minimize the link stress for a multipoint-to-point session. Algorithm EDRP balances the traffic load on the network fiber links. Apart from the primary objective, algorithm EDRP also tries to optimize the total cost as the second objective.

\subsection{Reverse Shortest Path Tree Routing Algorithm (RSPT)}

This algorithm solves the routing and wavelength assignment problem for a multipoint-to-point session separately. As shown in Algorithm 1, a shortest path tree SPT rooted at destination $d$ is first constructed. Then, the edges in the shortest path tree SPT are reversed in order to transmit light signals from each source node 
to destination $d$. After the search of routes, the wavelength assignment is implemented to satisfy the distinct wavelength constraint. Different wavelengths have to be employed for any pair of source nodes if their shortest paths to $d$ share at least a common link.

\section{$2.2 k$-Bounded Edge Disjoint Path Routing Algorithm (EDPR)}

From the point of view of the total cost, the Reverse Shortest Path Tree routing algorithm is able to provide the optimal solution. However, RSPT algorithm is not competitive for minimizing the link stress. RSPT algorithm only routes the multipoint-to-point traffic over the shortest paths, but it does not consider the relevant relation between the paths from all the sources to the same destination. Especially, some fiber link around the destination and leading to source nodes will be commonly used. Since different light signals are transmitted over each shortest path, the fiber links commonly shared by these shortest paths are highly loaded in the absence of costly optical traffic grooming switches [11]. The more traffic is concentrated on one fiber link, the more wavelengths will be required. Although sometimes we can not avoid the frequent usage of the links adjacent to the destination, we can at least balance the traffic among the fiber links adjacent to the destination. As the traffic load is balanced and evenly distributed, the maximum number of wavelengths needed in one fiber link (i.e., link stress) is reduced. Based on this fact, a $k$-Bounded Edge Disjoint Path Routing algorithm is proposed.

Different from the RSPT algorithm, the $k$-Bounded Edge Disjoint Path Routing algorithm considers the relationship between the lightpaths from all sources to the destination node when searching the routing paths. It aims to find the maximum edge disjoint paths for the source nodes. To achieve this, an edge removal action in the network topology is introduced during the lightpath computation. The edge removal action takes place whenever the lightpath for from a source node to destination $d$ is found. At the $j^{t h}$ step, the source $\tilde{\mathbf{s}}$ nearest to destination $d$ in renewed graph $G_{j}$ is added to lightstartree via the shortest path $S P_{G_{j}}(\tilde{\mathbf{s}}, d)$ such that it holds $\frac{S P_{G_{j}}(\tilde{\mathbf{s}}, d)}{S P(\tilde{\mathbf{S}}, d)} \leq k$ (refer to step-10 in Algorithm 2). To avoid using the same link twice, all the links in the lightpath specified for a source will be removed from the graph (refer to step-13). Then, the nearest source is computed in the renewed graph and the shortest path in the renewed graph is specified as the lightpath for the nearest destination. It is true that, most of the time, one light-startree is not sufficient to span all the sources. In

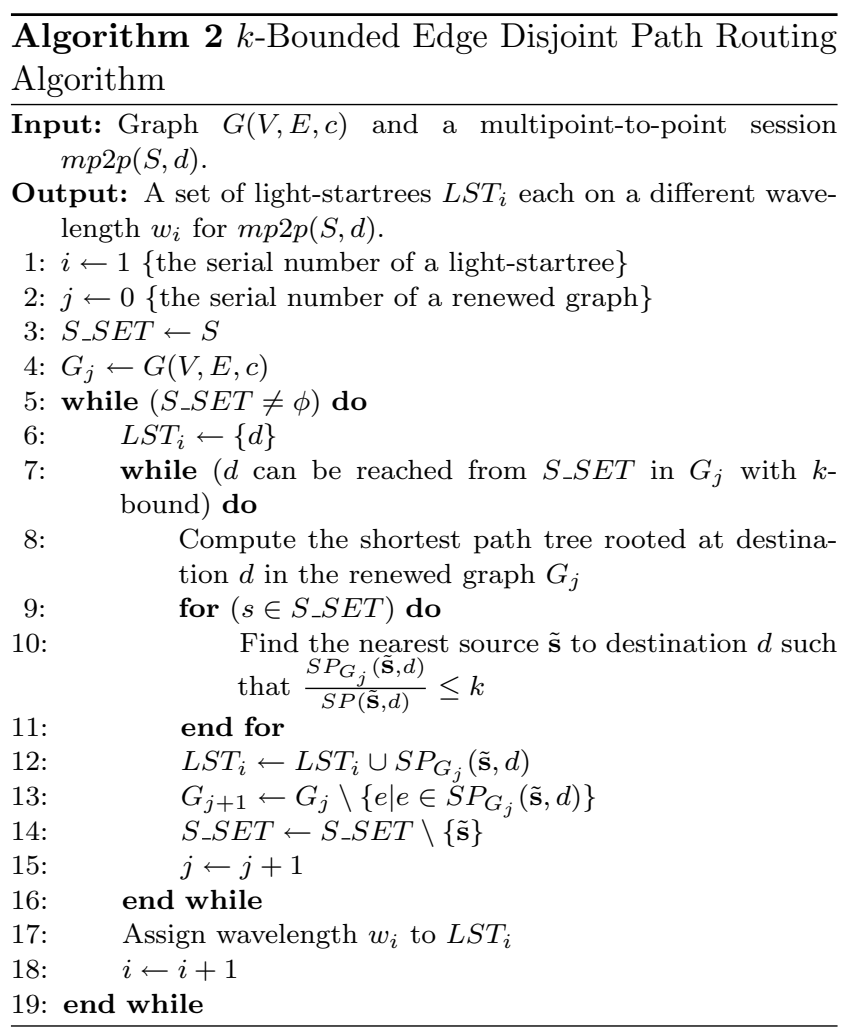

case that the destination is not reachable from the unspanned sources in the renewed graph, another lightstartree assigned a new wavelength will be computed over the initial topology using the same technique.

\subsection{An Example}

Given a topology in Fig. 2(a), multipoint-to-point session $m p 2 p\left(\left(s_{1}, s_{2}, s_{3}\right), d\right)$ is required. The number on each link signifies the link cost, for instance link $\left(d, s_{1}\right)$ has a cost of 1 . According to RSPT algorithm, a shortest path tree rooted at $d$ is first constructed. As shown in Fig. 2(b), the link $\left(d, s_{1}\right)$ is shared by three source nodes, and link $\left(s_{1}, s_{2}\right)$ is shared by $s_{1}$ and $s_{2}$. It is obvious that three light-startrees each on a different wavelength (i.e., $w_{0}, w_{1}$, and $w_{3}$ ) are needed. Thus, the link stress of RSPT algorithm is 3. However, if we apply EDPR algorithm, a favorable result will be obtained. First we find lightpath $L P\left(d, s_{1}\right)$ for the nearest source node $s_{1}$ to $d$ and add it to the light-startree. Then $L P\left(d, s_{1}\right)$ is removed from the topology. In the next step, it is observed that $s_{3}$ is the nearest source to $d$ in the renewed topology. The shortest path $L P\left(d, s_{3}\right)$ in the renewed topology is added to the light-startree. After that, $\operatorname{LP}\left(d, s_{3}\right)$ is also removed from the previous topology. Finally lightpath $\operatorname{LP}\left(d, s_{2}\right)$ is found. It is observed in Fig. 2(c) that EDPR algorithm just needs to construct one light-tree and thus only one wavelength 


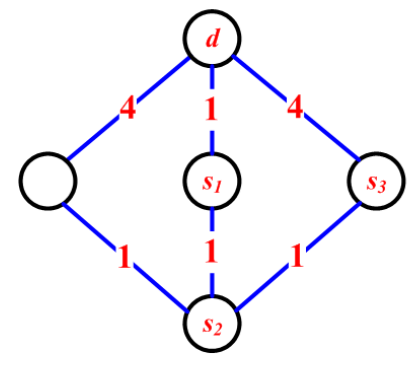

Topology

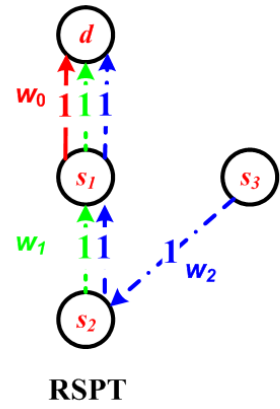

(b)

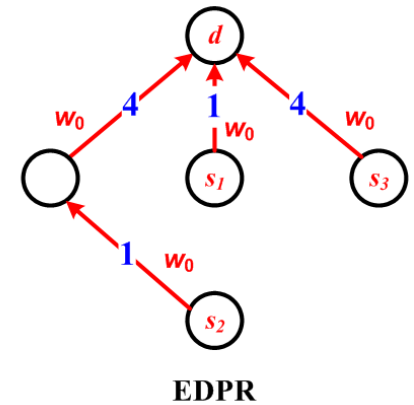

(c)

Fig. 2 (a) An example topology; (b) The result of RSPT algorithm; (c) The solution of EDPR algorithm.

is sufficient to cover all the multipoint-to-point session members. Hence, the link stress of EDPR algorithm is 1. We can also note that EDPR algorithm consumes 10 wavelength channel cost while RSPT algorithm utilizes only 6 wavelength channel cost.

\subsection{Discussion of Bound $k$}

The main objective of introducing the bound $k$ on the length of a path is to limit the total cost of a multipointto-point session $m p 2 p(S, d)$. The bound $k$ should be chosen carefully, since it greatly influences the quality of the computed light-startrees. Specifically, we consider two extreme cases:

(1) $k=1$. In this case, the candidate shortest path in the modified graph $G_{i}$ should satisfy $S P_{G_{i}}(s, d)=$ $S P(s, d)$. As a result, the potential path for a source $s$ to communicate with destination $d$ is strictly limited to the shortest path between $s$ and $d$ in the original network topology. This case corresponds exactly to the Reverse Shortest Path Tree routing algorithm. And the link stress is often very high in this case.

(2) $k=\infty$. In this case, there is no limitation on the length of the potential path from a source node $s$ to destination $d$. If this is the case, the EDPR algorithm can be interpreted as follows. At the $j^{\text {th }}$ iteration, the nearest source $s$ to destination $d$ in the renewed graph $G_{j}$ is added to the current light-startree. In fact, $k$ is not necessary equal to infinity. When $k$ is large enough, this property can also be guaranteed. The shortcoming of this method is that the total cost may be very big. A source node $s$ may connect to destination $d$ with a path traversing all the nodes in the network, which is not favorable at all.

From these two cases, we deduce that $k$ should be neither too small nor too large. It can be a practical method to determine a suitable $k$ by plotting the link stress and the total cost versus the bound $k$. And, dif- ferent values of $k$ may be employed for different network topologies.

\section{Overview of the Proposed Algorithms}

Theorem 1 RSPT algorithm provides a routing and wavelength assignment solution with the minimal total cost for a multipoint-to-point session $m p 2 p(S, d)$.

Proof Let us suppose $L$ light-startrees $L S T_{i}\left(S_{i}, d\right)$ are computed out for $m p 2 p(S, d)$, where $S_{i}$ exclusively denotes the leaf source nodes (or served source nodes) in this light-startree. For multipoint-to-point communications, only the leaf source nodes in a light-startree can be served in the absence of data aggregation. The other non-leaf source nodes in the light-startree are just used to forward the light signals to $d$ which are emitted by the source nodes in $S_{i}$. And it is entirely useless to serve the same source node several times in different light-startrees for the same multipoint-to-point communication. Hence, $\cup_{i=1}^{L} S_{i}=S$, and any pair of $S_{i}$ are disjoint. Moreover, a light-tree, in fact, is a set of edge disjoint lightpaths $L P(s, d)$ from each served source to $d$. Then, equations (1) can be rewritten as

$$
\begin{aligned}
c[m p 2 p(S, d)] & =\sum_{i=1}^{L} \sum_{e \in L S T_{i}\left(S_{i}, d\right)} c(e) \\
& =\sum_{i=1}^{L} \sum_{s \in S_{i}} \sum_{e \in L P(s, d)} c(e) \\
& =\sum_{s_{i} \in S} \sum_{e \in L P\left(s_{i}, d\right)} c(e)
\end{aligned}
$$

According to RSPT algorithm, each source node $s_{i}$ is connected to destination $d$ via the shortest path $S P\left(s_{i}, d\right)$. The total cost of the solution provided by RSPT algorithm is

$c(R S P T)=\sum_{s_{i} \in S} \sum_{e \in S P\left(s_{i}, d\right)} c(e)$ 
Since,

$\forall s_{i} \in S, \sum_{e \in S P\left(s_{i}, d\right)} c(e) \leq \sum_{e \in L P\left(s_{i}, d\right)} c(e)$

by combining equations (2) and (3) it is obviously true for any routing algorithm that

$c(R S P T) \leq c[m p 2 p(S, d)]$

Thus, RSPT has the optimal total cost.

Theorem 2 RSPT algorithm can route the multipointto-point session $m p 2 p(S, d)$ in the time of $O(|V| \log |V|+$ $|V|+|E|)$, where $|V|$ and $|E|$ denote the number of nodes and the number of edges in the network respectively.

Proof RSPT algorithm consists of the routing part and the wavelength assignment part. In the routing part, the Dijkstra's algorithm is generally used to compute the shortest path. The most recent complexity of Dijkstra's algorithm is $O(|V| \log |V|+|E|)$ [12]. In the wavelength assignment part, the fork nodes in the shortest path tree are searched and each branch of a fork node should be assigned a different wavelength. There are at most $|V|$ nodes in the shortest path tree, hence the search of fork nodes in the shortest path tree can be completed via a Bread First Search method in time of $O(|V|)$. Overall, RSPT algorithm can find the solution in $O(|V| \log |V|+|V|+|E|)$.

Theorem 3 Algorithm EDPR is able to achieve $k$-approximation in terms of total cost.

Proof In EDPR algorithm, source $s_{i}$ can be spanned to the current light-startree only if the shortest path $S P_{G_{i}}\left(s_{i}, d\right)$ in the modified graph $G_{i}$ satisfies the inequality below

$\sum_{e \in S P_{G_{i}}\left(s_{i}, d\right)} c(e) \leq k \times \sum_{e \in S P\left(s_{i}, d\right)} c(e)$

Through equation (2), the total cost of the light-startrees computed by EDPR algorithm for $m p 2 p(S, d)$ could be obtained by

$$
\begin{aligned}
c(E D P R) & =\sum_{s_{i} \in S} \sum_{e \in S P_{G_{i}}\left(s_{i}, d\right)} c(e) \\
& \leq k \times \sum_{s_{i} \in S} \sum_{e \in S P\left(s_{i}, d\right)} c(e)
\end{aligned}
$$

Hence,

$$
\frac{c(E D P R)}{c(R S P T)} \leq k
$$

As $c(R S P T)$ is the optimal total cost, EDPR achieve a cost approximation ratio of $k$.
Theorem 4 Algorithm EDPR solves the routing and wavelength assignment problem in the time of $|S| \times$ $O(|V| \log |V|+|E|)$.

Proof Algorithm EDPR realizes the routing and wavelength assignment for a multipoint-to-point communication at the same time. The sources are added to the light-startree iteratively. At each step, Dijkstra'algorithm is used to compute the nearest destination. Since one source is added at a time, $|S|$ iterations are required. All the light-startrees can be computed out in time of $|S| \times O(|V| \log |V|+|E|)$ for a session with $|S|$ sources.

Theorem 5 Let $D(d)$ be the degree of destination $d$ in graph $G$, and $w[m p 2 p(S, d)]$ denote the number of wavelengths required to accommodate the multipoint-topoint session $\operatorname{mp} 2 p(S, d)$. It holds true that:

$|S| \geq w[m p 2 p(S, d)] \geq \max \left\{\left\lceil\frac{|S|}{D(d)}\right\rceil, 1\right\}$

Proof Let $n_{i}$ be the number of lightpaths that traverses the $i^{\text {th }}$ link adjacent to destination $d$, then it satisfies

$\forall i \in[1, D(d)] \quad 0 \leq n_{i} \leq|S|$

Note that diverse light signals are carried on each lightpath $L P(s, d), n_{i}$ wavelengths are required in the $i^{t h}$ link adjacent to $d$. Since $|S|$ lightpaths should be established for a session, we obtain

$\sum_{1 \leq i \leq D(d)} n_{i}=|S|$

Link stress equals the maximum number of wavelengths needed per fiber, i.e.,

$w[m p 2 p(S, d)]=\max _{1 \leq i \leq D(d)} n_{i}$

First of all, at least one wavelength must be used for a multipoint-to-point session. If $\forall i$ and $j \in[1, D(d)], n_{i}$ $=n_{j}$, we can get

$\min \left\{\max _{1 \leq i \leq D(d)} n_{i}\right\}=\max \left\{\left\lceil\frac{|S|}{D(d)}\right\rceil, 1\right\}$

It means all $|S|$ lightpaths are distributed equally on the adjacent links of $d$. And it is obvious if $\exists k$ such that $n_{k}=|S|$, and $\forall i \in[1, D(d)]$ and $i \neq k, n_{i}=0$, we can obtain

$\max \left\{\max _{1 \leq i \leq D(d)} n_{i}\right\}=|S|$

It happens when all the lightpaths from $|S|$ source nodes to destination $d$ share the same link adjacent to $d$. 


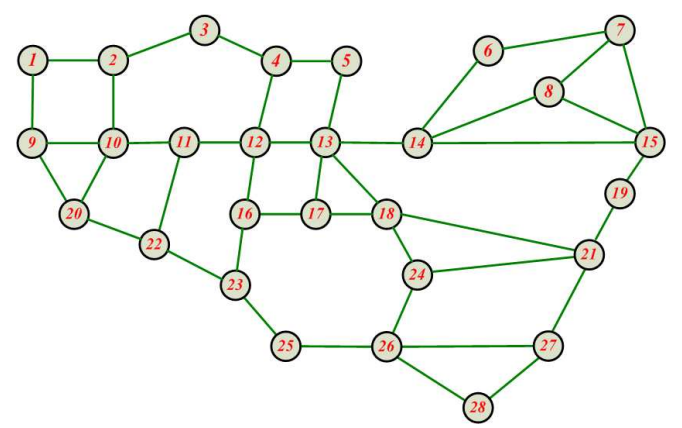

(a)

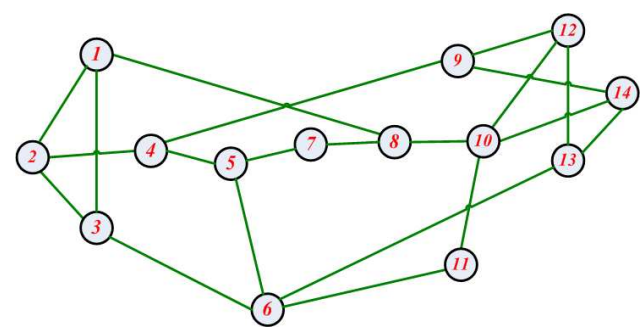

(b)

Fig. 3 Simulation Topologies (a) USA Longhaul Network (b) NSF Network.

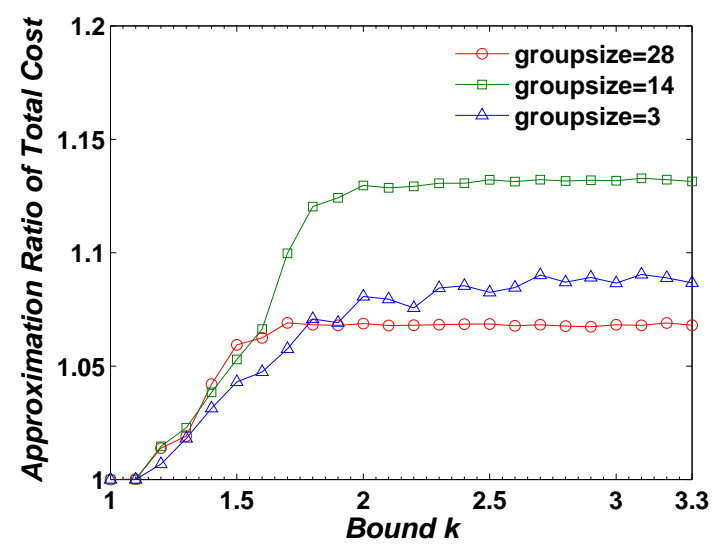

(a)

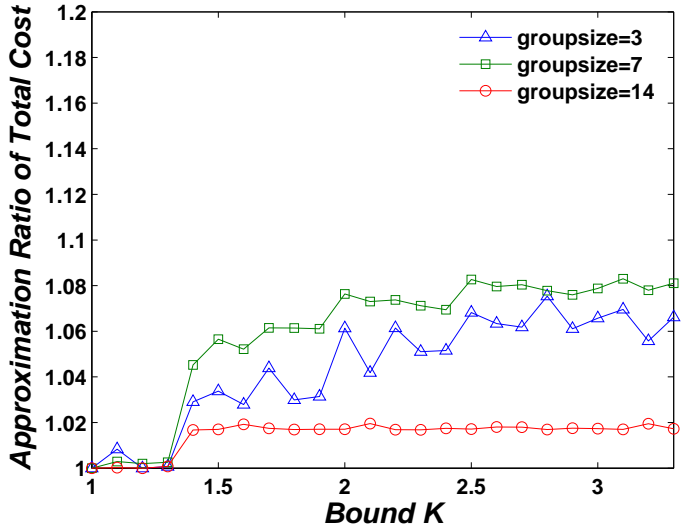

(b)

Fig. 4 Approximation Ratio of Total Cost versus Bound $k$ (a) in USA Longhaul network (b) in NSF Network

\section{Numerical Results}

To verify the theoretical analysis, extensive simulations are implemented to get numerical results. Both 28 nodes USA Longhaul network (refer to Fig.3(a)) and 14 nodes NSF Network (refer to Fig.3(b)) topologies are selected as the WDM network testbeds, since they are viewed as potential candidates for the future backbone network of USA and referenced in many literatures[1317]. An equal cost of 1 unit hopcount cost is assumed on all the links in the network to simplify the computations. In the simulation, destination and source nodes of each multipoint-to-point session are assumed to be distributed uniformly and independently in the topology. Given a group size, about 10000 sessions are generated, and thus each point in the curbs is the average of 10000 samples. Two metrics are considered to evaluate the quality of the computed light-startrees for a multi-point-to-point session $m p 2 p(S, d)$ :

- Total Cost: the total number of wavelength channels in the light-startrees constructed for $m p 2 p(S, d)$. It is calculated by equation (1) or (2).
- Link Stress: the maximum number of wavelengths required per link to span all the source nodes in $\operatorname{mp} 2 p(S, d)$.

In this section, first of all, the impact of the parameter $k$ in $k$-Bounded Edge Disjoint Path Routing algorithm (EDPR) is investigated. Then RSPT and EDPR algorithms are compared in terms of the aforementioned two metrics.

\subsection{Impact of Bound $k$ in EDPR Algorithm}

As stated in sub section 2.4, the bound $k$ should be chosen carefully, since it has a great impact on the performance of the algorithm. Here, we will study the quality of light-startrees when the bound $k$ changes, which could be a good reference for the selection of bound $k$. The EDPR algorithm with different bounds $k \in[1,3.3]$ is applied and simulated. The approximation ratio of total cost and the performance of link stress versus bound $k$ are plotted in Figs. 4 and 5, where the size of multipoint-to-point session is set to 3,7 and 14 for NSF network and 3, 14 and 21 for Longhaul network 


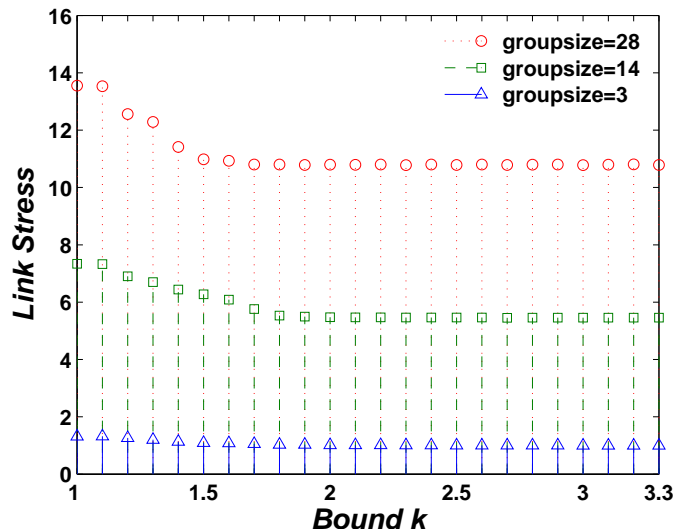

(a)

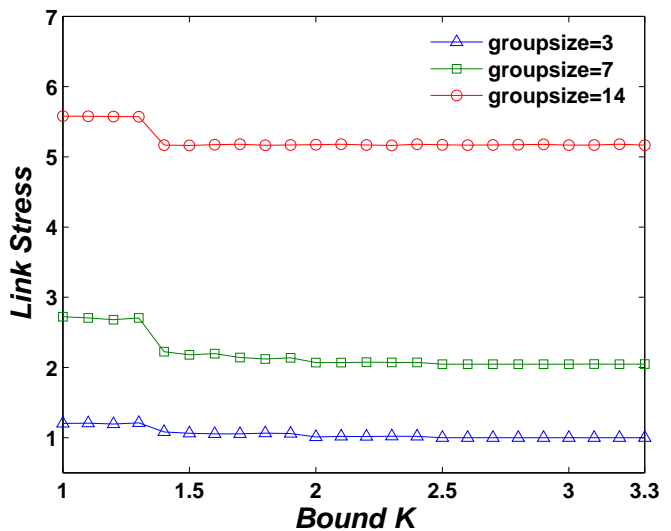

(b)

Fig. 5 Link Stress versus Bound $k$ (a) in USA Longhaul network (b) in NSF Network

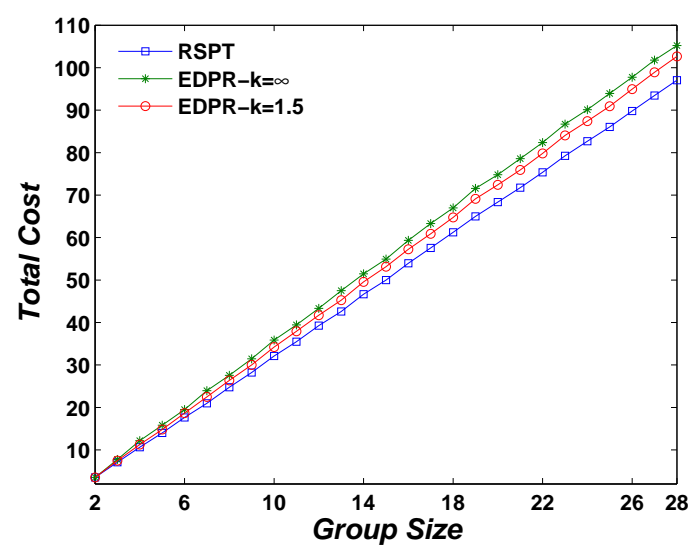

(a)

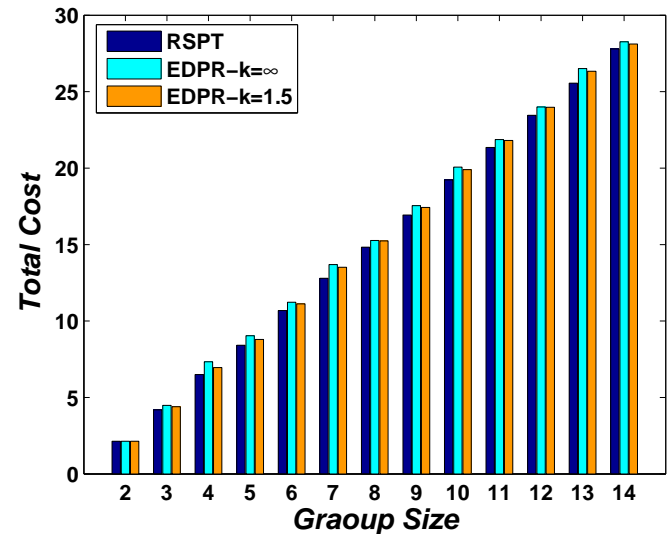

(b)

Fig. 6 Total Cost versus Multipoint-to-point Session Group Size (a) in USA Longhaul network (b) in NSF Network

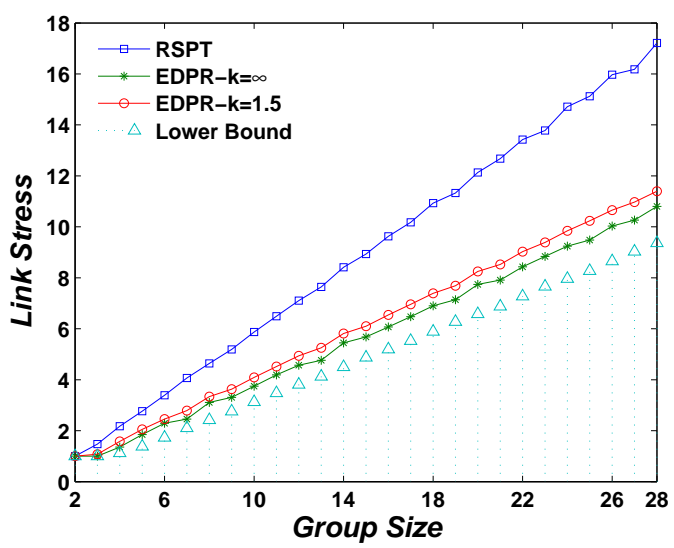

(a)

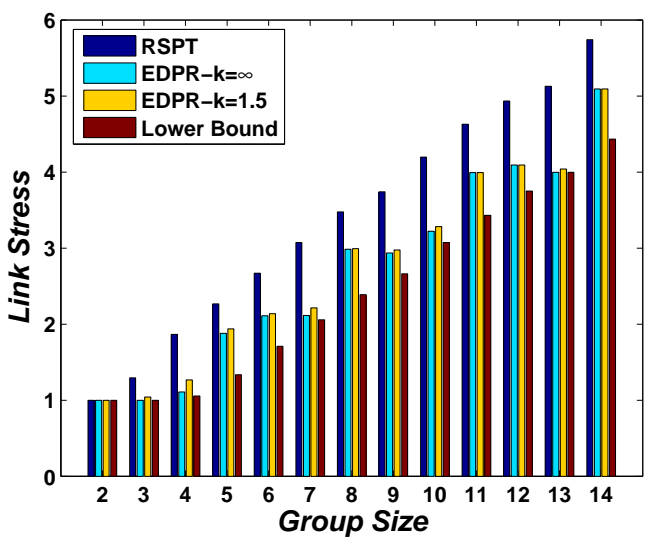

(b)

Fig. 7 Link Stress versus Multipoint-to-point Session Group Size (a) in USA Longhaul network (b) in NSF Network 
respectively (about corresponds to the member ratios of $20 \% / 10 \%, 50 \%$ and $100 \%$ ).

As indicated in both Figs. 4(a) and (b), the approximation ratio becomes worse as the the bound $k$ grows. This result exactly complies to the Theorem 3 . The bigger $k$ is, the more candidate paths will be found. Thus, we risk of utilizing longer paths. It is noted that the obtained approximation ratio in the simulation is always smaller than $k$, for instance the approximation ratio is 1.06 when $k=1.5$ in $4(\mathrm{a})$. This is because the approximation ratio $k$ given in Theorem 3 is the worst case ratio but not the average one. We can also see that, the approximation ratio stays stable when the bound $k$ increases beyond a certain value. In Longhaul network, the stable status begins when $k \geq 1$.8. And in NSF network although the approximation ratio in the curb with group size 3 fluctuates, we can see after $k \geq 2$ the approximation ratios in the curbs with group sizes of 7 and 14 achieve the highest value and keep almost stable. This result can be explained by the analysis of subsection 2.4. Because the diameter of a network is a fixed value (e.g., the diameter of USA Longhaul Network is 8 , and it is 3 for NSF Network), the value of $\frac{S P_{G_{j}}(s, d)}{S P(s, d)}$ is limited. Once $k$ increases above a certain value, EDPR algorithm can get the same total cost as that when $k$ is infinite.

The tendency of link stress versus the bound $k$ is shown in Figs. 5(a) and (b). We can find that the link stress of EDPR algorithm steps down rapidly at the beginning (when $k \leq 1.5$ ) as bound $k$ grows, then it goes down slowly. After the decreasing process, the link stress keeps steady. The EDPR algorithm can reach the best link stress when $k=1.8$ in Longhaul network (refer to Fig. 5(a)) and when $k=2.0$ in NSF network (refer to Fig. 5(b)). This phenomenon can be interpreted as follows. As $k$ increases, the condition of the possible candidate lightpaths from a source to the destination $d$ is relaxed. Then more possible lightpaths can be found for a source. However, similar to the situation of the the total cost, there will be restriction on the length of a possible lightpath if $k$ is large enough. Consequently, the link stress will be stable.

\subsection{Comparison of RSPT and $k$-Bounded EDPR}

In Figs. 6 and 7, the quality of light-startrees computed by RSPT and $k$-bounded EDPR algorithms are compared when the group size varies. As discussed in the previous part, in order to guarantee the cost approximation ratio, $k$ should not be too big. While a certain favorable small link stress should also be satisfied which prevent from choosing a too small $k$. Thus, we set $k=1.5$ in the simulation of this part, where the link stress finishes the rapid decreasing process and steps to the slow decreasing process. At this moment, the approximation ratio is about 1.05 in Longhaul network and 1.04 in average in NSF network. The comparisons are also done when $k=\infty$ and $k=1.5$ respectively in $k$-bounded EDPR algorithm.

Based on the simulation results in Figs. 6(a) and (b), the total cost races up rapidly when the multipoint-topoint session group size increases. The RSPT algorithm is able to get the best total cost which is also the optimal one as proved in Theorem $1 . k=1.5$-bounded EDPR algorithm get a favorable cost very near to that of RSPT in both Longhaul and NSF networks. $k=\infty$ bounded EDRP algorithm results in the highest total cost among the three. These results also coincide well with the discussion about $k$ in subsection 2.4 and the simulations results in Figs. 4.

The link stress against the group size is also demonstrated in Figs. 7(a) and (b). The lower bound of link stress is calculated by $\max \left\{\left\lceil\frac{|S|}{D(d)}\right\rceil, 1\right\}$, which is given in Theorem 5. According to the results, the link stress also grows up rapidly when the multipoint-to-point session group size increases. Althouht the RSPT algorithm achieves best total cost, its link stress is always the highest. $k=1.5$-bounded EDPR algorithm is able to get almost the same link stress as that of the $k=\infty$-bounded EDPR algorithm. Besides, their values are very close to the lower bound.

From all the simulation results in the previous two subsections, we can conclude that the bound $k$ influences not only the total cost but also the link stress. Given a multipoint-to-point communication, in order to assure some cost approximation ratio as well as some acceptable link stress simultaneously, $k$ should be chosen neither too big nor too small. In other words, a tradeoff should be found between the total cost and the link stress to satisfy different types of multimedia multipoint-to-point traffics.

\section{Conclusion}

Routing and wavelength assignment is a very important issue for supporting multipoint-to-point communications in all-optical WDM networks. Distinct wavelengths should be employed by source nodes to send light signals to the same destination $d$, if their lightpaths leading to $d$ are not edge disjoint. The lightstartree structure is introduced to route multipoint-topoint communications, which consists of several edge disjoint lightpaths assigned the same wavelength and terminated at the same destination. To establish a multi- 
point-to-point session, a set of light-startrees, each with a different wavelength, may be required. As the number of wavelengths supported in one fiber link is limited, not only the total cost but also the link stress are important parameters to measure the quality of lightstartrees computed. For this reason, the Reverse Shortest Path Tree routing algorithm is proposed, which is proven to be able to minimize the total cost in time complexity of $O(|V| \log |V|+|V|+|E|)$, where $|V|$ and $|E|$ denote the number of nodes and the number of edges in the network respectively. Meanwhile, the $k$-Bounded Edge Disjoint Path Routing algorithm is presented to route a multipoint-to-point session with as fewer wavelengths as possible. It results in significant reduction of the link stress. The total cost approximation ratio of the $k$-Bounded Edge Disjoint Path Routing algorithm to the optimal solution is given by $k$. Numerical results obtained by extensive simulations verify the efficiency of the two proposed routing and wavelength assignment algorithms in supporting all-optical multipoint-to-point communications.

\section{References}

1. George N. Rouskas. Optical layer multicast: rationale, building blocks, and challenges. IEEE Network, 17(1): 60-65, 2003.

2. Biswanath Mukherjee. Optical communication networking. McGraw-Hill, 1997.

3. Biswanath Mukherjee. WDM Optical communication networks: progress and challenges. IEEE Journal on Selected Aeras in Communications, 18(10): 1810-1824, 2000.

4. Laxman H. Sahasrabuddhe, Biswanath Mukherjee. Lighttrees: optical multicasting for improved performance in wavelength-routed networks. IEEE Communications Magazine, 37(2): 67-73, 1999.

5. R. Malli, Xijun Zhang, Chunming Qiao. Benefit of multicasting in all-optical networks. SPIE Proceeding on All-Optical Networking, 2531: 209-220, 1998.

6. Xijun Zhang, John Wei, Chunming Qiao. Constrained multicast routing in WDM networks with sparse light splitting. IEEE/OSA Journal of Lightware Technology, 18(12): 19171927, 2000.

7. Fen Zhou, Miklós Molnár, Bernard Cousin. Distance priority based multicast routing in wdm networks considering sparse light splitting. The $11^{\text {th }}$ IEEE International Conference on Communication System, pp709-714, Guangzhou, China, November, 2008.

8. Jianping Wang, Xiangtong Qi, Mei Yang. Routing and wavelength assignment for core-based tree in WDM networks. Computer Communications, 29: 1896-1904, 2006.

9. T. Billhartz, J.B. Cain, E. Farrey-Goudreau, B. Rieg, S.G. Batsell. Performance and resource cost comparisons for the CBT and PIM multicast routing protocols. IEEE Journal on Selected Areas in Communications 15(3): 304-315, 1997.

10. Osama Awwad, Ala I. Al-Fuqaha, Ammar Rayes. Traffic grooming, routing, and wavelength assignment in WDM transport networks with sparse grooming resources. Computer Communications, 30: 3508-3524, 2007.

11. Keyao Zhu, Hui Zang, Biswanath Mukherjee. A comprehensive study on next-generation optical grooming switches. IEEE
Journal on Selected Areas in Communications, 21(7): 11731186, 2003.

12. Michael Barbehenn. A note on the complexity of Dijkstra's algorithm for graphs with weighted vertices. IEEE Transaction on Computers, 47(2): 263, 1998.

13. Fen Zhou, Miklós Molnár, Bernard Cousin. Is light-tree structure optimal for multicast routing in sparse light splitting WDM networks. The $18^{t h}$ International Conference on Computer Communications and Networks, pp1-7, August 2, 2009, San Francisco, USA.

14. Fen Zhou, Miklós Molnár, Bernard Cousin. Supporting Multipoint-to-point Communicationas in All-Optical WDM Networks. The $34^{\text {th }}$ IEEE International Conference on Local Computer Network (LCN), pp209-212, Oct.19-23, 2009, Zürich, Switerland.

15. Oliver Yu, Yuan Cao. Mathematical formulation of optical multicast with loss-balanced light-forest. In proceeding of IEEE GLOBECOM, p1788-1792, 2005.

16. Fen Zhou, Miklós Molnár, Bernard Cousin. Avoidance of Multicast Incapable Branching Nodes in WDM Netwoks. Photonic Network Communications, 18(3):378-392, 2009. Springer.

17. Ashraf M. Hamad, Ahmed E. Kamal. Optical amplifier placement in WDM mesh networks for optical multicasting service support. IEEE/OSA Journal of Optical Communications and Networking, 1(1):85-102, 2009. 